\title{
Foresight must guide geoengineering research and development
}

To the Editor - Large-scale climate geoengineering will probably be required to limit warming to well below $2{ }^{\circ} \mathrm{C}$, in line with the 2015 Paris Agreement ${ }^{1}$. While geoengineering has long been included in the development of many (future) lowcarbon emissions pathways ${ }^{1}$, key questions remain about the efficacy and ability of such climate intervention to fulfil its anticipated role in limiting warming ${ }^{2,3}$. As such, the social license for geoengineering has not been granted for research using unbounded large-scale experiments, never mind fullscale deployment.

Given the urgency of finding solutions to mitigating climate change, a pathway is needed to jointly achieve efficacy and social license. However, the increasing impatience by some proponents to begin field experiments in geoengineering, supported by public and private investment and philanthropy, appears to assume that social licence will be granted in response to science excellence alone. Furthermore, if such planned pilot studies are not done in a rigorous and transparent manner, with sufficient due diligence, they have the potential to damage and in some cases shut down promising avenues of research into geoengineering. Parallels can be drawn with the suite of ocean iron-enrichment experiments $\left(\sim 1,000 \mathrm{~km}^{2}\right)$ designed to simulate changes to ocean productivity and the enhanced carbon sequestration recorded in the geological record ${ }^{4}$. These experiments revealed surprises and artefacts such as their poor representation of many oceanic processes ${ }^{4}$. In turn, this suite of experimental findings led to both research governance and the need to explore fitfor-purpose designs of larger scale studies $\left(100,000 \mathrm{~km}^{2}\right)\left(\right.$ ref. $\left.^{5}\right)$. The international research governance was developed through amendments to the London Convention ${ }^{6}$. At present, a range of planned geoengineering pilot studies have the potential to set a dangerous precedent for others to follow with their own independently regulated experiments ${ }^{7}$.
There are at least four proposed pilot studies on geoengineering that have been publicised recently: Marine Cloud Brightening Project, Ice 911, Sky River and SCoPEx. In particular, SCoPEx (the Stratospheric Controlled Perturbation Experiment), planned for the southwestern United States later this year, has yet to publish fit-for-purpose model simulations of the exact scale, extent and area of experimentation, required to satisfy broad public concerns around this research ${ }^{8}$. Overlooking this step is particularly troubling given that solar radiation management experiments have the potential, even at seemingly small scales, to perturb or encroach on the air space of other states and nations, potentially leading to conflict ${ }^{9}$.

We concur with the viewpoint that "Research in climate engineering must proceed - even if it turns out to be unnecessary" ${ }^{10}$. However, given the spectrum of technological, social, science and governance issues associated with geoengineering, along with lessons learnt from $>10$ large-scale ocean iron-enrichment studies, it is essential to first elucidate the longer-term goals and then identify the trajectory towards a full implementation. This trajectory must be formulated around the scientific and engineering questions relevant for any geoengineering technique at each stage of its development. Equally important is that at every stage such a trajectory considers the needs for appropriate and agreed governance, and equally the requirements for granting of the social license to operate. Without this, the potential for unintended and widespread impacts may occur, particularly where there is a tension between the urgency to conduct such potentially pioneering research, and the correspondingly important need to achieve internationally agreed research governance.

As part of this foresighting, there is a strong requirement for (scale- and processappropriate) modelling studies to develop these trajectories from research towards implementation. Critically, this development must precede field experimentation to enable exploration of the wide array of issues around what a pilot study might reveal. To inform the development of realistic trajectories, fit-for-purpose modelling simulations must also look well beyond a pilot study, so as to uncover the characteristics of the subsequent suite of more advanced and upscaled experiments with respect to efficacy, side-effects, and detection and attribution. This line-of-sight from modelling will enable researchers to clearly examine and address the implications of the trade-offs between running pilot experiments with or without agreed global research governance (for example, C2G2; ref. ${ }^{11}$ ) and the implications for the granting of a social license to operate in this increasingly contentious space.

\section{Andrew Lenton ${ }^{1,2 *}$, Philip W. Boyd ${ }^{2,3}$, Marcus Thatcher ${ }^{4}$ and Kathryn M. Emmerson ${ }^{4}$ \\ ${ }^{1}$ CSIRO Oceans and Atmosphere, Hobart, Tasmania, Australia. ${ }^{2}$ Antarctic Climate and Ecosystems Co- operative Research Centre, University of Tasmania, Hobart, Tasmania, Australia. ${ }^{3}$ Institute for Marine and Antarctic Studies, University of Tasmania, Hobart, Tasmania, Australia. ${ }^{4}$ CSIRO Oceans and Atmosphere, Aspendale, Victoria, Australia. *e-mail:andrew.lenton@csiro.au}

Published online: 22 April 2019 https://doi.org/10.1038/s41558-019-0467-z

\footnotetext{
References

1. Rogelj, J. et al. Nature 534, 631-639 (2016).

2. Lawrence, M. G. et al. Nat. Commun. 9, 3734 (2018).

3. Irvine, P. et al. Nat. Clim. Change 9, 295-299 (2019).

4. Boyd, P. W. et al. Science 315, 612-617 (2007).

5. Watson, A. J., Boyd, P. W., Turner, S. M., Jickells, T. D. \& Liss, P. S Mar. Ecol. Prog. Ser. 364, 303-309 (2008).

6. 1996 Protocol to the Convention on the Prevention of Marine Pollution by Dumping of Wastes and Other Matter, 1972 (IMO, 1996); http://www.imo.org/en/OurWork/Environment/LCLP/ Documents/PROTOCOLAmended2006.pdf

7. Tollefson, J. Nature 490, 458-459 (2012).

8. Tollefson, J. Nature 563, 613-615 (2018).

9. Chhetri, N. et al. Governing Solar Radiation Management (Forum for Climate Engineering Assessment, American University, 2018). 10. Nature 516, 8 (2014)

11. Carnegie Climate Geoengineering Governance Initiative (C2G2, 2019); https://www.c2g2.net/the-need-for-governance/
} 